MATEC Web of Conferences 48,05005 (2016)

DOI: $10.1051 /$ matecconf $/ 20164805005$

(C) Owned by the authors, published by EDP Sciences, 2016

\title{
Sensory Dissociation in Vestibular Function Assessment
}

\author{
Ivan Tolmachev ${ }^{1, a}$, Konstantin Brazovsky ${ }^{2}$, Sergey Schadenko ${ }^{2}$ and Ekaterina Korzhenkova ${ }^{1}$ \\ ${ }^{1}$ National Research Tomsk Polytechnic University, 634050 Tomsk, Russia \\ ${ }^{2}$ Siberian state medical university, 634050 Tomsk, Russia
}

\begin{abstract}
The project aims to create a solution to the problem of early diagnostics of neurodegenerative disorders, accompanied by imbalance, and to develop rehabilitation methods for patients with vestibular disorders. The balanced upright position is a result of interaction between vestibular system, skeleton and muscles, visual, and proprioceptive systems. Postural abnormalities are caused by morphological or functional alteration of the components of the equilibrioception system. These disorders weaken the ability of the central nervous system to process vestibular, visual and proprioceptive signals, which are responsible for the sense of balance. Moreover, it also decreases the plasticity of the nervous system, resulting in adaptation disfunctionality. Unfortunately, premorbid detection of vestibular dysfunctions is quite a complicated test because available methods to evaluate postural reflexes have low sensitivity and specifity. Consequently, the development of methods to assess functional state of the equilibrioception system becoming viable in order to detect neurodegenerative disorders as early as possible, to control treatment and rehabilitation procedures.
\end{abstract}

\section{Introduction}

Application of sophisticated computer methods for medical diagnostics, as well as for scientific research and education became the common practice these days. Modern automated complexes allow obtaining research data quickly and with a high confidence level. Unfortunately, increase of the volume of information to process doesn't lead, automatically, towards boost of diagnostics quality and precision. The point is that the obtained raw data characterizing the state of the complex multilevel biological system at the specified moment of time. However, the system state itself is not the essential indicator of biological system ability to adapt and function normally. We are proposing the convenient approach towards correlation of measured parameters of biosystem with the needed values. The approach is based on integrated evaluation of condition and allows to consolidate heterogeneous multidimensional data into the integrated criteria, which characterizing the rate of system deviation from some reference state (e.g. norm, average value, etc.). It was shown that the proposed approach could be applied for data of different nature (e.g. infrared spectra of absorption spectrum of exhaled air, tomographic images, electro-physiological measurements, etc.). The only limitation of the approach is the necessity to have powerful computational resources, to model the states of biosystems using varied set of data. However, the problem could be solved by utilizing the parallel and highperformance computing. During the analysis of dynamic processes in biology and medicine in general, and in the diagnostics of human movement, in particular, the large amount of data is

\footnotetext{
${ }^{a}$ Corresponding author : ivantolm@mail.ru
} 
generated. Posturology is a relatively new trend in neurology. This branch focuses on the development and use of various assessment methods and approaches for the diagnostics of disorders of human balance system. One method to evaluate the functional state of the vestibular system is to record the behaviour of the overall pressure centre on the surface with a force platform. This method, called stabilometry, is actually the "gold standard" in postural studies. Nevertheless, it has several drawbacks, for example, fairly large dimensions of the equipment that must be installed properly and carefully calibrated before use. Usage of the high-performance computing environment allows to create a multi-parametric evaluation system of movement quality and functional equilibrium state of a human, which includes a set of techniques, such as dynamic stabilometry, video capturing and detection of movements, electroencephalography, oculography, myography. Meanwhile, in recent years, due to the development of computer graphics, we have an opportunity to create a virtual threedimensional visual environment, which simulates the actual environment. The virtual environment could be easily controlled via a computer to simulate the desired motion and thus to carry out detailed studies of various aspects of visual information in the postural control. Postural control of the patient without vestibular disorders is realized through the visual signals. The virtual environments can cause a conflict between the perception of movement, created by the visual system with the vestibular and proprioceptive input signals, indicating a static state. This vestibular-visual and proprioceptive conflict of the "neural redundancy" is weakened or even completely absent, if there is an acute sensory deprivation, which affects postural control. We assume that the postural studies during the process of specific impact of the virtual environment could allow assessing postural strategy and the role of each signal stream: visual, vestibular and proprioceptive. In terms of diagnostics, such approaches can detect the process of adaptation of the human balance system, hence allows detecting the presence of a compensatory process at an early stage, enhancing the function of the visual analyzer in maintaining the vertical position of the person.

Postural disorders may be connected with diseases of various organs and tissues, which affect the normal functioning and determine the clinical manifestations and prognosis. Causes may be different: injuries, birth defects and predispositions, influence of food, environmental and genetic factors, or idiopathic reasons [1]. All these disorders affect the central and the peripheral nervous system, and the musculoskeletal system, disrupting the balance and posture of the individual [2].

One of the most frequent causes of balance disorders and postural instability is Parkinson's disease [3]. Parkinson's disease is a neurodegenerative disease that affects the central nervous system [4, 5, 6], and is clinically characterized by slowness of movement, resting tremor, rigidity, postural disorders and a number of non-motor symptoms. The etiology of this disease is still unknown; however, the main cause of Parkinson's disease is the loss of dopaminergic cells primarily in the nigrostriatal system [7], which leads to movement disorders.

Parkinson's disease is a progressive disorder, and during the disease the motor defect becomes more pronounced, which explains the emergence and intensification of symptoms such as resting tremor, rigidity, bradykinesia, changes in posture, instability, impaired balance and walk [8]. Moreover, this disease decreases the ability of the central nervous system to process vestibular, visual and proprioceptive impulses responsible for maintaining balance. Therefore, Parkinson's disease causes reduction of the ductility and as result loss of adaptive reflexes [9]. It should be noted that the population of people with vestibular disorders and the population of patients with Parkinson's disease might be at least partially overlap because pharmacokinetics and pharmacodynamics of antiparkinsonian drugs promotes labyrinth symptoms [10]. Thus, a correlation between Parkinson's disease, disorders of the vestibular system and physical balance shows the importance of the study and balance evaluation of patients with Parkinson's disease.

Objective: development and application of virtual environment for virtual reality devices Oculus Rift DK 2, for assessment of the impact of neurological tests in a virtual reality system to maintain balance of patients with Parkinson's disease. 


\section{Methods of study}

Virtual three-dimensional visual environment is a room decorated in neutral black with bright orange lines and boundaries between floor-wall-ceiling (Fig. 1). This design does not have any distraction, which allows for assessment of the functional state of the system to maintain the equilibrium of the human body.

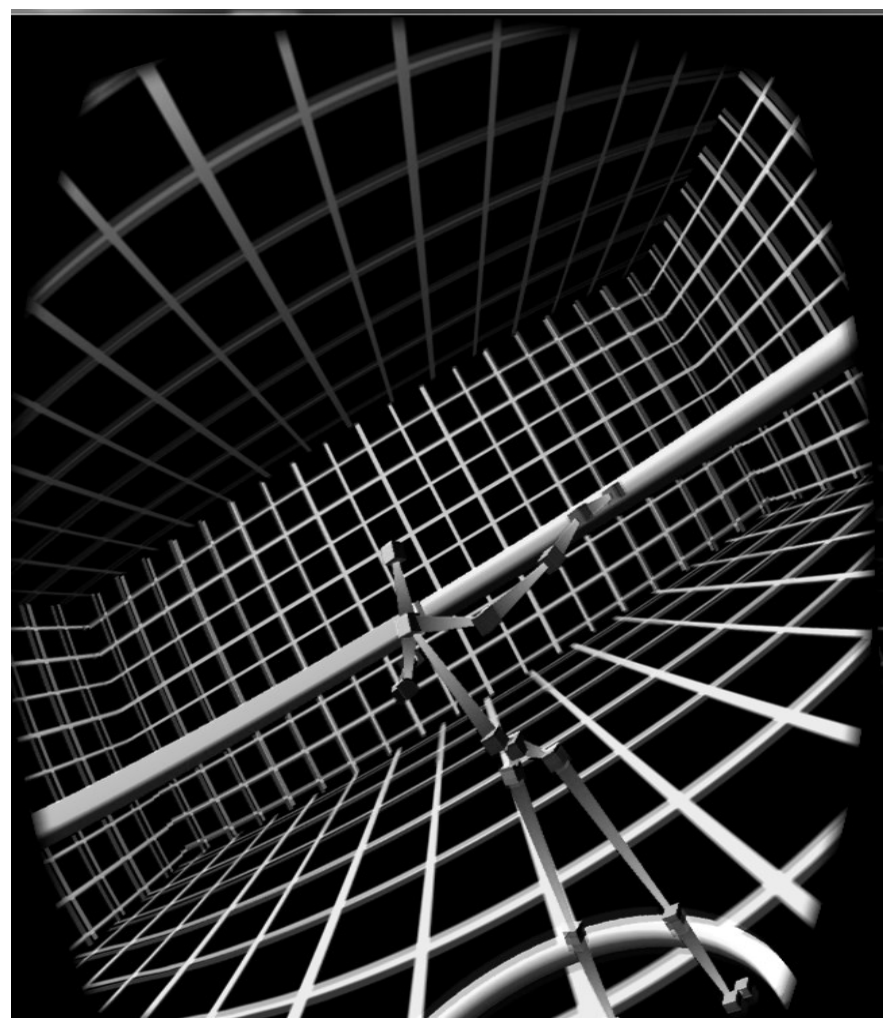

Figure 1. Three-dimensional environment for assessment of the impact of neurological tests in a virtual reality.

Scenario studies of the vestibular apparatus using virtual reality was designed. To study the possibilities of the proposed method were conducted quantitative estimates of motion vectors when performing routine tests Romberg in the study of neurological status in virtual reality for 20 seconds. The movement data of the body were recorded using markerless motion capture technology. This technique allows recording the three-dimensional coordinates of 20 standard points on the body with a frequency of 15 samples per second. The study involved 12 volunteers without disorders of the balance function; in the second group was 10 subjects with Parkinson's disease. The study of each test included several stages. Stage without glasses: eyes open, eyes closed. Stage with virtual reality glasses: tracking label to the left at 90 degrees for $10 \mathrm{sec}$., Tracking label to the right at 90 degrees for $10 \mathrm{sec}$., Tracking label up at $1.75 \mathrm{~m}$. for $10 \mathrm{~s}$., Tracking label down at $1.75 \mathrm{~m}$. for $10 \mathrm{sec}$., tilt the room forward on 30 degrees, tilt the room back on 30 degrees, tilt the room to the left on 30 degrees, tilt the room to the right on 30 degrees. Evaluation of the path of the points of the body was carried out using the method of integral estimates. The theoretical details of the criterion were published in [13].

At the first step. Motion vector fragment skeleton model as a set of coordinates $(t, x, y z)$ is formed. The whole interval $[0, \mathrm{~T}]$ then is divided into $\mathrm{k}$ fragments with length $\Delta \mathrm{T}$. The average values $x j, j=0, \ldots, K$ are calculated inside the $[t, t+\Delta T]$ interval. The average values are normalized and stored in a matrix $\mathrm{N} \times \mathrm{k}$. Where $\mathrm{N}$ is a number of subjects in a certain group. Calculation distancebased criterion based on the calculation of the Mahalanobis distance. The theoretical details of the criterion were published in [13]. For the calculation of distance-based criterion Id we have developed 


\section{MATEC Web of Conferences}

a computer program. Input data are files that contain the normalized values of the reference and tested condition.

\section{Conclusion}

When the Romberg test with open eyes is provided, in both cases there was an impact on the visual analyzer. But in the first case, it was a virtual visual environment while the second - a real room. Compared to the stage of testing with closed eyes the results of these studies showed a smaller deviation integral criterion.

Results of the study of neurological functional tests with closed eyes and using a virtual reality in a group of healthy humans have shown that variations in the point corresponding to the centre of mass were significantly reduced in comparison with the right and left hands. Even so, results of the study of subjects with Parkinson's disease have shown increasing variations in the point corresponding to the centre of mass.

In addition, the characteristics of the obtained values increased integral criterion in the range of 414 seconds. The reaction of the receptor cells of the vestibular apparatus, caused by a change in body position in space or a movement leads to a redistribution of reflex muscle tone. Consequently, the time interval from the start of the sample until the maximum value of the integral criterion can be the time of reflex reactions skeletal muscles that maintain the body balance.

\section{References}

1. O. S. Levin, N. V. Fedorova, Parkinson's disease, 352 (2012)

2. R. Stephenson, A. Siderowf, M. B. Stern, Mov. Disord. 24(2), 665 (2009)

3. Y. Miki, F. Mori, K. Wakabayashi, N. Kuroda, S. Orimo, Mov. Disord. 24, 2299 (2009)

4. C. Gaig, E. Tolosa, Mov. Disord. 24, 656 (2009)

5. J. Jankovic, J Neurol Neurosurg Psychiatry 79, 368 (2008)

6. K. J. Brusse, S. Zimdars, K. R. Zalewski, T. M. Steffen, Phys Ther. 85(2), 134 (2005)

7. B. N. Smetanin, G. V. Kozhin, Neurophysiology. 39(6), 476 (2007)

8. T. Mergner, G. Schweigart, C. Maurer, A. Blumle, Exp. Brain Res. 167(3), 535 (2005)

9. V. A. Fokin, Bulletin of the Tomsk Polytechnic University 311(5), 120 (2007) 